\title{
Modification of Semi-Analytical Method Applied System of ODE
}

\author{
OPhir Nave ${ }^{1}$ \\ ${ }^{1}$ Department of Mathematics, Jerusalem College of Technology, Jerusalem, Israel \\ Correspondence: OPhir Nave, Department of Mathematics, Jerusalem College of Technology, Jerusalem, Israel.
}

Received: May 2, 2020

doi:10.5539/mas.v14n6p75
Accepted: May 23, 2020

Online Published: May 26, 2020

URL: https://doi.org/10.5539/mas.v14n6p75

\begin{abstract}
In this study, we modify the well-known semi-analytical method called the Homotopy Analysis Method (HAM), such that the right-hand side of a given ODE system is decomposed to a sum of analytical functions. We called the new semi-analytical method: decomposition of the Homotopy Analysis Method (DHAM). We applied the new method to a breast cancer mathematical model. We compared the DHAM results to HAM and numerical simulations. We concluded that the DHAM results are closer to the numerical simulation results than the HAM.
\end{abstract}

Keywords: semi analytical method, homotopy analysis method (HAM), breast cancer mathematical model

\section{Introduction}

\subsection{Introduce the Problem}

In this section we introduce the information according to ICA (Israel Cancer Society, 2020).

Breast cancer is an abnormal growth of the breast lobe cell or mammary duct. These cells grow uncontrollably and may spread to other areas of the body. Both women and men can get breast cancer, although breast cancer in men is rare. Breast cancer is the most common malignant disease in Israel and the Western world in general. About 5,000 women are diagnosed with breast cancer in Israel each year. If the disease is diagnosed in the early stages, the chances of healing increase to about $90 \%$. Evidently, the number of women recovering from breast cancer in Israel is steadily rising, thanks to early detection improved treatment and widespread awareness.

Causes of breast cancer:

The causes of breast cancer are not yet fully known, but there are several factors that increase the risk of illness. It is important to note that even if a woman has one or more risk factors, she does not necessarily have cancer; similarly as absence of risk factors does not guarantee that she will not have cancer.

- Sex:

Breast cancer is far more common in women than in men - statistically, there is less than one male patient for every 100 female patients. According to the National Cancer Registry, about 75 men are diagnosed with breast cancer every year, compared to more than 5,400 women.

- Age:

The risk of getting cancer increases with age. Data from the National Cancer Registry in Israel found that breast cancer is more common in women aged 50 and over, and about $78 \%$ of patients are diagnosed at these ages. About $15 \%$ of patients are diagnosed between the ages of $40-49$ and about $6 \%$ of patients are diagnosed under the age of 40 .

- Family history:

Women historical family of breast cancer or ovarian cancer, especially if they have a first-degree relationship (mother or sister), or women who have been diagnosed with a genetic mutation that increases the risk of getting sick (such as BRCA1 or BRCA2 gene mutations), are more likely to have breast cancer.

- Medical history and breast structure:

A woman who has previously had breast cancer or pre-malignant conditions such as DCIS, (Atypical Ductal) LCIS, (Ductal Carcinoma In Situ Hyperplasia, non-cancerous breast disease), is more at risk of getting breast cancer. Additionally, women with dense breast structure are at higher risk - the term dense breast refers to a situation where the most breast tissue is made up of connective tissue and very little fat tissue. This situation is 
particularly characteristic of young women.

- Radiation therapy (radiotherapy) for the chest area at an early age:

A woman who has undergone radiation therapy to the chest (usually as a treatment for Hodgkin's lymphoma), especially at the age of breast development, is more at risk for breast cancer.

- Hormonal factors that increase the risk of breast cancer:

Women taking hormone replacement therapy (HRT) to treat menopausal symptoms, especially estrogen and progesterone combination therapy, for over 10 years. When treatment is stopped, the risk decreases. These women are advised to keep a close medical record and take the treatment for as short a time as possible.

Women who never gave birth, or gave birth at an older age, after the age of 35 .

Women who did not breastfeed at all or breastfeed for less than a year.

Women who started their menstrual cycle at a young age (before age 11) or entered menopause at a relatively older age (after age 55).

- Lifestyle:

In scientific studies, there is a clear correlation between increased risk of breast cancer and obesity, lack of exercise, improper nutrition, increased alcohol consumption, and smoking.

Many researchers such as physicists and mathematicians, in addition to medical professionals, study cancer. As with all phenomena in the natural sciences, researchers try to create mathematical models that will accurately describe the considered phenomenon. The problem is that there are many factors in the human body that interact with diseases in general and cancer in particular, for example the immunotherapy research area. Immunotherapy is an anti-cancer treatment method that helps the body's immune system identify and attack cancer cells. Immunotherapy is part of a group of therapies called Targeted Therapy, or Targeted or Biological, which uses the patient's immune system to attack the tumor cells more effectively. Unlike chemotherapy treatment, which acts on all body cells that divide at a rapid rate, whether malignant or not, immunotherapeutic drugs act on cell-specific molecules related to cancer, such as PD1 and CTLA4.

Because many immune cells are involved, it is very difficult to formulate a mathematical model that takes into account all the parameters of the tumor's interaction with the cells. Hence, all models that exist in the literature today are approximate models. Usually, these models include a non-partial differential equation system. Sometimes these models are non-ordinary differential equation systems, as we will present in this article. The common methods to investigate these models are the application of numerical simulations. In this study, we applied the well-known semi-analytical method called the Homotopy Analysis Method (HAM). We modify this method such that the right-hand side of the mathematical model is decomposed into a sum of analytical functions. This method is called Decomposition Homotopy Analysis Method (DHAM).

The significant advantage of the HAM method is that it is not necessary to present the mathematical model in the form of a Singular Perturbed System (SPS). The HAM method artificially inserts a small parameter into the mathematical model and thus the model can be investigated using various asymptotic methods.

\section{Method}

In this section we describe in detail the Homotopy Analysis Method and the modification of this method.

\subsection{HAM Method}

In this section we present the concept of HAM as introduced in (Liao, 2009) and (Liao, 2010). Since the model we are investigating is time-dependent only, the method of homotopy will be written not in general but in the way it fits in the model under consideration. Consider the following system of differential equations:

$$
\mathcal{N}_{i}\left[u_{i}(t)\right]=g_{i}(t), \quad 1 \leq i \leq k,
$$

where $\mathcal{N}_{i}$ are nonlinear operator, $t$ denotes time and $u_{i}$ are unknown functions, and $g_{i}(t)$ are known analytic functions. For $g_{i}(t)=0$, the system (1) are the homogeneous system.

The zero-order deformation equation is defined as:

$$
(1-p) \ell\left[\Phi_{i}(t ; p)-u_{i, 0}(t)\right]=p \hbar_{i} \mathcal{N}_{i}\left[\Phi_{i}(t ; p)-g_{i}(t)\right]
$$

where $\hbar_{i}$ is a non-zero auxiliary parameter called convergence-control parameter, $\ell$ is an auxiliary linear operator, $u_{i, 0}(\cdot)$ is an initial guess of $u_{i}(\cdot)$, and $\Phi_{i}$ are unknown functions. The degree of freedom is to choose the initial guess, the auxiliary linear operator, and the auxiliary parameter. If setting $p=0$ and $p=1$ 
at equation (2) one has: $\Phi_{i}(t ; p=0)=u_{i, 0}(t)$ and $\Phi_{i}(t ; p=1)=u_{i}(t)$ respectively. Thus as $p$ increases from 0 to 1 the solution $\Phi_{i}(t ; p)$ varies from the initial guesses $u_{i, 0}(t)$ to the solutions $u_{i}(t)$.

Expanding $\Phi$ in Taylor series with respect to the embedding parameter $p$, one has

$$
\Phi_{i}(t ; p)=u_{i, 0}(t)+\sum_{n=1}^{\infty} u_{i, n}(t) p^{n},
$$

where

$$
u_{i, n}(t)=\left.\frac{1}{n !} \frac{\partial^{n} \Phi_{i}(t ; p)}{\partial p^{n}}\right|_{p=0} .
$$

If the auxiliary linear operator, the initial guess, and the auxiliary parameter, are so properly chosen that the above series converges at $p=1$, and one has

$$
\Phi_{i}(t ; 1)=u_{i, 0}(t)+\sum_{n=1}^{\infty} u_{i, n}(t),
$$

which must be one of the solutions of the original nonlinear equation.

The zero-order deformation is a private case of mth-order deformation as follows: define the vector:

$$
\vec{u}_{i, n}(t)=\left\{u_{i, 0}(t), u_{i, 1}(t), \ldots, u_{i, n}(t)\right\}, \quad 1 \leq i \leq k \quad(k=13) .
$$

Differentiating Equation (2) $m$-times with respect to the embedding parameter $p$ and then setting $p=0$ and finally dividing the terms by $m$ !, we obtain the $m$ th-order deformation equation in the form of:

$$
\ell\left[u_{i, m}(t)-\chi_{m} u_{i, m-1}(t)\right]=\hbar_{i} R_{i, m}\left(\vec{u}_{i, m-1}\right),
$$

where,

$$
R_{i, m}\left(\vec{u}_{i, m-1}\right)=\left.\frac{1}{(m-1) !} \frac{\partial^{m-1} \mathcal{N}_{i}\left[\Phi_{i}(t ; p)-g_{i}(t)\right]}{\partial p^{m-1}}\right|_{p=0},
$$

and $\chi_{m}$ is the unit step function. Applying the inverse operator $\ell^{-1}(\cdot)$ on both side of Equation (6), we get

$$
u_{i, m}(t)=\chi_{m} u_{i, m-1}(t)+\hbar_{i} \ell^{-1}\left[R_{i, m}\left(\vec{u}_{m-1}\right)\right] .
$$

In this way, it is easy to obtain $u_{i, m}$ for $m \geq 1$, at $m$ th-order and finally obtain the solution as:

$$
u_{i}(t)=\sum_{n=0}^{m} u_{i, n}(t) .
$$

In our model we choose the initial guess to be the initial conditions of the considered model.

The linear operator will be:

$$
\ell=\frac{d}{d \tau}(\cdot)
$$

\subsection{Decomposition of Homotopy Analysis Method}

In this section we present the new concept of the DHAM method. Given a system of differential equations

$$
\mathcal{N}_{i}\left[u_{i}(t)\right]=g_{i}(t), \quad 1 \leq i \leq k .
$$

The base assumption of the DHAM method is that the function $g_{i}(t)$ can be divided into $n$ terms such as

$$
g_{i}(t)=f_{i}^{0}(t)+f_{i}^{1}(t)+\ldots+f_{i}^{n}(t) .
$$

Expanding $g_{i}(t)$ in powers of the embedding parameter $p$ as:

$$
g_{i}(t)=\phi_{i}(t ; p)=f_{i}^{0}(t) p^{0}+f_{i}^{1}(t) p^{1}+\ldots+f_{i}^{n}(t) p^{n},
$$

then the zero-order deformation in this case has the form of:

$$
(1-p) \ell\left[\Phi_{i}(t ; p)-u_{i, 0}(t)\right]=p \hbar_{i} \mathcal{N}_{i}\left[\Phi_{i}(t ; p)-\phi(t)\right]
$$

where $\phi=\sum_{i} \phi_{i}$.

The $m^{\text {th }}$ order deformation has the form of:

$$
\ell\left[u_{i, m}(t)-\chi_{m} u_{i, m-1}(t)\right]=\hbar_{i} R_{i, m}\left(\vec{u}_{i, m-1}\right),
$$

where

$$
R_{i, m}\left(\vec{u}_{i, m-1}\right)=\left.\frac{1}{(m-1) !} \frac{\partial^{m-1} \mathcal{N}_{i}\left[\Phi_{i}(t ; p)-\phi_{i}(t)\right]}{\partial p^{m-1}}\right|_{p=0} .
$$

\section{The Mathematical Model of Breast Cancer}

In this section, we present our mathematical model of breast cancer that includes the initiation, progression, and recurrence after conservative breast surgery and the effect of radiotherapy on preventing local recurrence. The equations for the mathematical model describe stepwise mutations that change a normal breast stem cell to a tumor cell. The main assumptions of the model are as follows (Heiko, at. al., 2007): 
- Two genes (Tumor Suppressor Genes) $\boldsymbol{T S G}$ s are sufficient to give rise to a tumor.

- The dynamics of the system describe a linear mutation pathway, the mutations have to occur in a certain order, and mutations in $\boldsymbol{T} \boldsymbol{S} \boldsymbol{G}_{\mathbf{2}}$ can only occur after $\boldsymbol{T} \boldsymbol{S} \boldsymbol{G}_{\mathbf{1}}$ is deactivated.

- The dynamic variables of the system do not distinguish whether mutation in the paternal allele occurs first and is followed by the mutation in the maternal allele, or vice versa (i.e., $\boldsymbol{T} \boldsymbol{S} \boldsymbol{G}^{-/+}$or $\boldsymbol{T S G}^{+/-}$),

- The model takes into account two target alleles for the first mutation in a gene, compared to only one for the second mutation. The two $\boldsymbol{T} \boldsymbol{S} \boldsymbol{G}_{\mathrm{s}}$ are labelled as $\boldsymbol{T} \boldsymbol{S} \boldsymbol{G}_{\mathbf{1}}$ and $\boldsymbol{T} \boldsymbol{S} \boldsymbol{G}_{\mathbf{2}}$. When both alleles are wild-type, they are

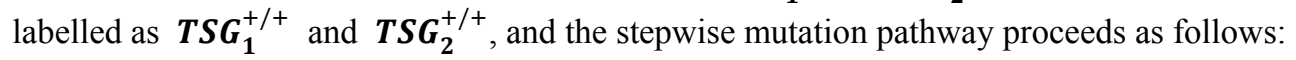

$$
\begin{aligned}
& T S G_{1}^{+/+} T S G_{2}^{+/+} \stackrel{\mathrm{p}_{1}}{\rightarrow} T_{S G_{1}^{+/-}} T_{S G_{2}^{+/+}}^{+\mathrm{p}_{2}} \rightarrow T_{S G_{1}^{-/-}} T_{S G_{2}^{+/+}} \stackrel{\mathrm{p}_{3}}{\rightarrow} \\
& T S G_{1}^{-/-} T S G_{2}^{+/-} \stackrel{\mathrm{p}_{4}}{\rightarrow} T S G_{1}^{-/-} T_{S G_{2}^{-/-},}
\end{aligned}
$$

where $T S G_{1,2}^{+/-}$represents a $T S G$ with loss of heterozygosity $(L O H)$ and $T S G_{1,2}^{-/-}$represents an inactivated $T S G . p_{i}$ is the probability that one allele in a normal cell mutates, and at the final step of the pathway when both of the TSGs are inactivated, the cell becomes cancerous.

The dynamic variables of the system are:

$\boldsymbol{f}$ - breast tissue with a fraction of healthy breast stem cells $\left(T S G_{1}^{+/+}{ }^{2} S G_{2}^{+/+}\right)$,

$\boldsymbol{q}$ - cells with $L O H$ in $T S G_{1}$,

$\boldsymbol{r}-T S G_{1}$ inactivated,

$\boldsymbol{s}-\mathrm{LOH}$ on $T S G_{2}$,

$\boldsymbol{n}$ - the production of tumor cells,

$\boldsymbol{m}$ - the production of enzymes,

$\boldsymbol{\nabla}$ - differential operator $\nabla=\left(\frac{\partial}{\partial x_{1}}, \ldots, \frac{\partial}{n}\right)$,

$\boldsymbol{\nabla}^{2}=\boldsymbol{\nabla} \cdot \boldsymbol{\nabla}$ where $\cdot$ is the scalar product,

Using the above assumptions, the mathematical model includes four nonlinear ordinary differential equations plus two partial differential equations:

$$
\begin{gathered}
\frac{d f}{d t}=-\kappa_{f} m f-\theta f, \\
\frac{d q}{d t}=\mu_{q} q\left(A_{\max }-A\right)-\kappa_{q} n q+\theta f-p_{2} q, \\
\frac{d r}{d t}=\mu_{r} r\left(A_{\max }-A\right)-\kappa_{r} n r+p_{2} q-p_{3} r, \\
\frac{d s}{d t}=\mu_{s} s\left(A_{\max }-A\right)-\kappa_{s} n s+p_{3} r-p_{4} s, \\
\frac{\partial n}{\partial t}=\mu_{n} n\left(A_{\max }-A\right)+d_{n} \nabla^{2} n-\chi \nabla \cdot(n \nabla f)+p_{4} s \\
\frac{\partial m}{\partial t}=d_{m} \nabla^{2} m+\xi n\left(1-m / m_{0}\right)-\omega m .
\end{gathered}
$$

The initial conditions of the model are as follows: we assumed that no mutations were established pre-puberty, therefore the whole breast tissue is made up of healthy $T S G_{1}^{+/+} T S G_{2}^{+/+}$cells. With the proposed mutation rates per gene per cell division given by $p_{i}$ and an assumed $5 \%$ of the healthy breast tissue representing breast stem cells which divide 50 times per year, the probability of developing a tumor within $40-60$ years is very small.

\section{Discussion}

We compared our analysis between the DHAM with HAM and numerical results. In order to determine the socalled optimal convergence control parameter we compute the following derivative (Liao, 2012):

$$
\frac{\partial\left(R e_{m}(\hbar)\right)}{\partial \hbar}=0
$$

where

$$
\operatorname{Re}(\hbar)=\int_{\Gamma}[\mathcal{N}(f(\tau))]^{2} d \tau
$$

and $\Gamma$ is the whole region of interest, $\mathcal{N}$ is the non-linear operator.

To compute the optimal of $\hbar$ one can apply different mathematics software such as MATHEMATICA 8, 
MAPLE, and so on. We concluded that for our calculations, the optimal value of the convergence control parameter is $\hbar=0.0023$. We insert this value of the parameter to the model that is rewritten in the mathematical model in HAM and DHAM form and run the algorithms of these two methods. For each method, we obtained the homotopy series which is an approximation of the real solution of the dynamical variables of the considered system. We solve the mathematical model using ode45 MATLAB for our comparative analysis.

In order to validate the DHAM method as well as the HAM method we define the absolute error for each method compared to numerical results as:

$$
\begin{aligned}
E r_{i}^{H A M} & =\left|u_{i, \text { numerical }}-u_{i, n}^{H A M}\right| \\
E r_{i}^{\text {DHAM }} & =\left|u_{i, \text { numerical }}-u_{i, n}^{D H A M}\right|,
\end{aligned}
$$

where

$$
u_{i, n}(t)=u_{i, 0}+u_{i, 1}+\ldots+u_{i, n}
$$

and the relative error to the numerical results in percent as

$$
\begin{gathered}
\operatorname{Er}_{i}^{H A M}(\%)=\frac{\left|u_{i, \text { numerical }}-u_{i, n}^{H A M}\right|}{u_{i, \text { numerical }}} \cdot 100 \%, \\
\operatorname{Er}_{i}^{\text {DHAM }}(\%)=\frac{\left|u_{i, \text { numerical }}-u_{i, n}^{\text {DHAM }}\right|}{u_{i, \text { numerical }}} \cdot 100 \%,
\end{gathered}
$$

The results are presented in Tables 1-4.

Table 1. The relative error (in percent) and the absolute error for the dynamical variable $\boldsymbol{f}$

\begin{tabular}{ccccc}
\hline$t$ & $E r_{i}^{H A M}$ & $E r_{i}^{\text {DHAM }}$ & $\operatorname{Er}_{i}^{\text {HAM }}(\%)$ & $\operatorname{Er}_{i}^{\text {DHAM }}(\%)$ \\
\hline 2 & $1.546 \cdot 10^{-4}$ & $0.229 \cdot 10^{-6}$ & 4.6 & 2.0 \\
4 & $2.879 \cdot 10^{-5}$ & $0.001 \cdot 10^{-8}$ & 4.5 & 0.1 \\
6 & $2.564 \cdot 10^{-6}$ & $0.434 \cdot 10^{-8}$ & 4.8 & 0.2 \\
8 & $4.875 \cdot 10^{-6}$ & $0.432 \cdot 10^{-8}$ & 8.7 & 0.1 \\
10 & $6.897 \cdot 10^{-7}$ & $1.232 \cdot 10^{-8}$ & 6.0 & 0.1 \\
12 & $7.876 \cdot 10^{-8}$ & $2.758 \cdot 10^{-10}$ & 3.1 & 2.3 \\
14 & $4.341 \cdot 10^{-9}$ & $2.585 \cdot 10^{-12}$ & 1.6 & 0.0 \\
16 & $2.463 \cdot 10^{-9}$ & $0.267 \cdot 10^{-13}$ & 0.6 & 0.0 \\
\hline
\end{tabular}

Table 2. The relative error (in percent) and the absolute error for the dynamical variable $\boldsymbol{q}$

\begin{tabular}{ccccc}
\hline$t$ & $E r_{i}^{H A M}$ & $E r_{i}^{\text {DHAM }}$ & $\operatorname{Er}_{i}^{\text {HAM }}(\%)$ & $\operatorname{Er}_{i}^{\text {DHAM }}(\%)$ \\
\hline 2 & $1.794 \cdot 10^{-6}$ & $1.423 \cdot 10^{-8}$ & 4.3 & 1.0 \\
4 & $2.255 \cdot 10^{-5}$ & $0.458 \cdot 10^{-8}$ & 4.4 & 1.2 \\
6 & $2.474 \cdot 10^{-6}$ & $0.766 \cdot 10^{-8}$ & 3.4 & 1.3 \\
8 & $3.255 \cdot 10^{-6}$ & $0.547 \cdot 10^{-7}$ & 1.2 & 0.2 \\
10 & $1.255 \cdot 10^{-8}$ & $2.685 \cdot 10^{-9}$ & 1.3 & 0.1 \\
12 & $0.474 \cdot 10^{-8}$ & $0.658 \cdot 10^{-10}$ & 0.3 & 0.0 \\
14 & $0.469 \cdot 10^{-9}$ & $0.995 \cdot 10^{-13}$ & 0.4 & 0.0 \\
16 & $0.432 \cdot 10^{-11}$ & $1.347 \cdot 10^{-15}$ & 0.5 & 0.0 \\
\hline
\end{tabular}


Table 3. The relative error (in percent) and the absolute error for the dynamical variable $\boldsymbol{r}$

\begin{tabular}{ccccc}
\hline$t$ & $E r_{i}^{H A M}$ & $E r_{i}^{\text {DHAM }}$ & $\operatorname{Er}_{i}^{\text {HAM }}(\%)$ & $\operatorname{Er}_{i}^{\text {DHAM }}(\%)$ \\
\hline 2 & $2.675 \cdot 10^{-3}$ & $0.876 \cdot 10^{-8}$ & 1.0 & 0.3 \\
4 & $2.765 \cdot 10^{-3}$ & $9.872 \cdot 10^{-8}$ & 1.2 & 0.1 \\
6 & $3.876 \cdot 10^{-4}$ & $0.767 \cdot 10^{-9}$ & 1.1 & 0.3 \\
8 & $4.655 \cdot 10^{-4}$ & $0.634 \cdot 10^{-10}$ & 1.2 & 0.2 \\
10 & $3.355 \cdot 10^{-5}$ & $7.342 \cdot 10^{-13}$ & 1.4 & 0.0 \\
12 & $2.7685 \cdot 10^{-5}$ & $1.437 \cdot 10^{-16}$ & 1.0 & 0.0 \\
14 & $4.876 \cdot 10^{-7}$ & $1.695 \cdot 10^{-18}$ & 0.3 & 0.1 \\
16 & $2.876 \cdot 10^{-8}$ & $1.238 \cdot 10^{-19}$ & 0.5 & 0.0
\end{tabular}

Table 4. The relative error (in percent) and the absolute error for the dynamical variable $\boldsymbol{s}$

\begin{tabular}{ccccc}
\hline$t$ & $E r_{i}^{H A M}$ & $E r_{i}^{D H A M}$ & $E r_{i}^{H A M}(\%)$ & $\operatorname{Er}_{i}^{D H A M}(\%)$ \\
\hline 2 & $0.231 \cdot 10^{-4}$ & $2.545 \cdot 10^{-6}$ & 0.3 & 0.0 \\
4 & $1.544 \cdot 10^{-4}$ & $2.555 \cdot 10^{-5}$ & 0.2 & 0.2 \\
6 & $2.478 \cdot 10^{-4}$ & $3.234 \cdot 10^{-8}$ & 1.2 & 0.3 \\
8 & $1.377 \cdot 10^{-4}$ & $0.457 \cdot 10^{-9}$ & 1.4 & 1.1 \\
10 & $1.589 \cdot 10^{-4}$ & $0.324 \cdot 10^{-12}$ & 3.3 & 1.2 \\
12 & $1.588 \cdot 10^{-4}$ & $0.646 \cdot 10^{-15}$ & 4.5 & 0.2 \\
14 & $0.388 \cdot 10^{-4}$ & $0.456 \cdot 10^{-18}$ & 0.2 & 0.0 \\
16 & $0.590 \cdot 10^{-4}$ & $0.333 \cdot 10^{-21}$ & 0.1 & 0.1 \\
\hline
\end{tabular}

To summarize the above study: We investigated a mathematical model that describes breast cancer progression. The mathematical model as presented does not have an explicit hierarchy, and therefore different asymptotic methods cannot be applied.

We therefore applied the method of homotopy analysis method that does not require an explicit hierarchy of the studied model, i.e. the model must not be of the SPS form. The method of homotopy artificially inserts a small parameter into the system of equations.

We proposed improving the method of homotopy by writing the right-hand part of the mathematical model with a sum of analytical functions.

We found the controlling parameter for the convergence of the results obtained from the methods of the homotopy analysis method.

Finally, we solved the mathematical model with the optimal parameter by order of 28 iterations of homotopy deformation. At the same time, we solved the considered model with numerical simulations. In order to validate the HAM method and the DHAM, we have defined a relative error and absolute error of the solutions with respect to the numerical solutions. We have concluded that for the relative error as well as the absolute error, the DHAH method is smaller in comparison to the HAM method.

\section{References}

ICA: Israel Cancer Society 2020, https://www.cancer.org.il/.

Liao, S. J. (2009). Notes on the homotopy analysis method: some definitions and theorems. Communications in Nonlinear Science and Numerical Simulation, 14(4), 983-997, https://doi.org/10.1016/j.cnsns.2008.04.013.

Liao, S. J. (2010). An optimal homotopy-analysis approach for strongly nonlinear differential equations. Communications in Nonlinear Science and Numerical Simulation, 15(8), 2003-2016, https://doi.org/10.1016/j.cnsns.2009.09.002

Liao, S.J. (2012). Homotopy analysis method in nonlinear differential equations. Springer/ New-York. https://doi.org/10.1007/978-3-642-25132-0_6 
Heiko, E., Mark, A.J.C, Alexander, R.A.A. \& Jayant, S.V. (2007). A mathematical model of breast cancer development, local treatment and recurrence. Journal of Theoretical Biology, 246, 245-259, https://doi.org/10.1016/j.jtbi.2006.12.010

\section{Copyrights}

Copyright for this article is retained by the author(s), with first publication rights granted to the journal.

This is an open-access article distributed under the terms and conditions of the Creative Commons Attribution license (http://creativecommons.org/licenses/by/3.0/). 